\title{
Preoperative preparation for elective laparoscopic cholecystectomy using spinal anesthesia: a case report
}

\begin{abstract}
Introduction: We describe the awake laparoscopic surgery using a high spinal blockade performed in a severe pulmonary cripple with alpha-1 antitrypsin deficiency. We then discuss the unique peri-operative care and anesthesia used to reduce the cardio-pulmonary stress in high risk patient populations

Case presentation: A 59year old Caucasian female presented with a history of asthma, alpha-1-antitrypsin deficiency, extensive panacinar emphysema and twenty years of symptomatic cholelithiasis. The FEV1/FVC ratio was $16 \%$ of predicted. Despite severe COPD, the patient demonstrated excellent functional status and was determined to be ASA Class 3. She desired intervention, but declined intubation unless absolutely indicated. Therefore, she was referred to an advanced minimally invasive surgical center to evaluate all possible options by convening a multidisciplinary team. We formulated a multi-tiered anesthesia plan to perform her cholecystectomy under high spinal analgesia with minimal pneumoperitoneum. The procedure was executed successfully utilizing very low pneumoperitoneum.

Conclusion: Preoperative preparation was a critical component in the successfu outcome of this case. Multidisciplinary preoperative assessment by both the surgical and anesthesia teams was followed by a group discussion with the patient. Consensus was reached regarding risk stratification, contingency planning, realistic expectations and perioperative safeguards. Introducing trocars into the abdominal cavity with minimal pneumo-peritoneum proved to be difficult and required troubleshooting. Adequate pain control was an integral aspect in the success of this procedure. This report serves as a guideline for those interested in providing similar services at institutions with limited prior experience.
\end{abstract}

Keywords: spinal anesthesia, minimally invasive surgery, laparoscopic cholecystectomy, multidisciplinary, preoperative planning
Volume 3 Issue I - 2016

\section{Brett C Parker,' Terrence R Burns, ${ }^{2}$ Steven D Schwaitzberg'}

'Department of Surgery, University at Buffalo, USA

2Department of Anesthesiology, University at Buffalo, USA

Correspondence: Brett C Parker, University at Buffalo, 149 Swan St., Apt. 203, Buffalo, NY, I 4203, USA, Tel (229) 254-3।8I, Email brettparker86@gmail.com

Received: December 13, 2015 | Published: February 16, 2016

\section{Introduction}

Spinal anesthesia has been well documented as a safe alternative for patients undergoing laparoscopic surgery with contraindications to general anesthesia. ${ }^{1,2}$ Medications infused directly into the cerebrospinal fluid provide sensory, motor and sympathetic blockade which can decrease cardiopulmonary stress in high risk surgical patients ${ }^{3}$ For patients with severe chronic obstructive pulmonary disease, laparoscopic cholecystectomy with low pressure pneumoperitoneum has been shown to be a cost effective, safe and effective approach. ${ }^{4-6}$ We describe the first laparoscopic case performed at Buffalo General Medical Center using a high spinal blockade, a multidisciplinary approach to patient preparation and troubleshooting port placement to ensure adequate exposure.

\section{Case presentation}

A 59year old Caucasian female presented with history of asthma, hypothyroidism, alpha-1-antitrypsin deficiency, extensive panacinar emphysema and twenty years of symptomatic cholelithiasis. A right upper quadrant ultrasound demonstrated numerous small stones and sludge. Liver function tests were within normal limits. Preoperative pulmonary testing was $16 \%$ of predicted values, yet she demonstrated excellent functional status. Because of her pulmonary status we formulated a multi-tiered plan to perform her surgery under high spinal analgesia with minimal pneumoperitoneum. The patient reported that at the time of her preoperative evaluation her gallbladder disease was in a quiescent phase and that her nutritional status was optimal. She had suffered two significant biliary colic attacks in the previous three months and was fearful she would require an emergent operation necessitating intubation in general anesthesia.

On the day of surgery the patient weighed $53 \mathrm{~kg}$ and was determined to be ASA Class 3. Prior to entry to the operating room, the patient received an albuterol ipratropium nebulizer treatment, one liter of warm crystalloid, $100 \mathrm{mg}$ of intravenous hydrocortisone and $1 \mathrm{~g}$ of intravenous acetaminophen. Spinal anesthesia was administered at the L2-L3 interspace with the patient in the sitting position. At the end point of the injection the patient was quickly positioned supine and maintained in a $15^{\circ}$ Trendelenburg with $15^{\circ}$ right lateral tilt for five minutes. A Foley catheter was not placed. The patient received an infusion of low dose intravenous dexmedetomidine and ketamine, with intermittent doses of midazolam and fentanyl. Oxygen was delivered at $1 \mathrm{~L}$ per minute via nasal cannula. After successful testing for adequate anesthesia, the abdomen was prepped with Betadine and draped with an iodine-impregnated incision drape. $30^{\circ}$ reverse Trendelenburg was then initiated. A vertical incision was made in the umbilicus and we directly opened the peritoneum. A balloon port was placed and the abdomen was insufflated slowly to $8 \mathrm{mmHg}$. A $10 \mathrm{~mm}$ 
subxiphoid port and two $5 \mathrm{~mm}$ lateral ports were placed under direct visualization. An unusually large and elongated gallbladder was found. An excellent critical view of safety was ultimately obtained, the cystic duct and artery were secured with hemoclips, and the gallbladder was then dissected from the liver bed using electro surgery.

The specimen was placed in a bag retrieval system and removed through the umbilicus. The procedure took approximately ninety minutes. Throughout the case, all vital signs were stable and oxygen saturation was $>97 \%$. Blood loss was minimal and the patient underwent straight catheterization at the end of the procedure to empty her bladder. Postoperatively, the patient was recovered in the surgical intensive care unit overnight as per the formulated plan and reported no abdominal pain or change in her respiratory status. She was discharged to her family home on postoperative day one with no sequelae. She returned to her home state by commercial aircraft one week later after a satisfactory postoperative evaluation. Subsequent telephone consultation revealed that she had completely returned to her normal activity by two weeks post surgery.

\section{Discussion}

Preoperative preparation was a critical component in the successful outcome of this case. Surgical and anesthesiology team members first had an opportunity to evaluate the patient in clinic, and then participated in a conference with the patient one week prior to admission. The patient shared insight on the nature of her lung disease, including precipitating and alleviating factors. At this time it was stressed that maintenance of spontaneous breathing was critical, as positive pressure ventilation would lead to gas trapping and V/Q mismatch. Together a three tiered plan was formulated, which would begin with spinal anesthesia, and escalate to general anesthesia via laryngeal mask airway or endotracheal intubation as needed.

The anesthesia team that evaluated the patient in clinic managed her in the operating room. We selected an experienced nursing team. We confirmed the availability of redundant supplies and equipment. Due to her acute on chronic disease, we anticipated the possibility of a shortened duct, and prepared for an intraoperative cholangiogram. Introducing trocars into the abdominal cavity with minimal pneumoperitoneum proved to be difficult. The abdominal wall did not have sufficient counter-tension to grant accurate port placement, resulting in scything of the instrument and ultimately increased case difficultly. To overcome this issue, some techniques describe increasing the abdominal pressure $>10 \mathrm{mmHg}$ while gaining access, then reducing the pneumoperitoneum. We avoided this approach because this would impair the patient's ability to ventilate. Instead, the first laparoscopic instrument placed into the abdominal compartment was used to create counter pressure on the abdominal wall to aid in accurate trocar placement for the remaining ports.

Laparoscopic procedures with reduced pneumoperitoneum also decrease the space in which the surgeon may operate. Previous studies demonstrate that placing the fourth port lower than usual at the level of the umbilicus may ease retraction of the gallbladder and improve exposure while maneuvering through an unusually small working space. ${ }^{4}$ Additionally, in cases of significantly enlarged gallbladders, using this fourth port location to grasp the gallbladder near the lower margin of the liver also made dissection easier. ${ }^{4}$ In the future, a single port laparoscopic approach may be considered for patients with small body habitus.
In addition to reduced pneumoperitoneum with intraperitoneal instrument assisting, there are many abdominal lift instruments on the market today that can be used to perform minimal invasive surgery on the high risk patient, elderly, pregnant women or children. These methods utilize subcutaneous point tenting to introduce the trocars under direct vision after the lift device is in place. Low pressure and gasless laparoscopy has been utilized and researched by this author in the past with good outcomes. ${ }^{7}$ The original Laparolift system was commonly used by this attending surgeon in the 1990's. More recently, the Storz Abdo-Lift device is being combined with low pressure $(3-4 \mathrm{mmHg})$ or gasless laparoscopy to gain access in these patient populations. However, in our experience these systems routinely lead to prolonged set up times and offered inadequate exposure. Furthermore, the constant abdominal wall tension required for these devices created unwarranted postoperative pain. Additionally, they are not commonly available in most surgical centers which make mastery of their use difficult.

Pain control was also a major aspect of this procedure. Her spinal anesthesia successfully controlled her pain throughout the immediate postoperative period, with no abdominal pain or sensation reported. Use of non-opioid anesthetic adjuncts was a mainstay of her anesthesia care. She reported increased tenderness in her shoulder during sub diaphragmatic irrigation with room temperature crystalloid. Such feedback from an awake patient is useful, prompting us to use warm irrigant in subsequent cases of this type. The surgical and anesthesia teams, in conjunction with a pre-notified pulmonary service, formed a vital partnership with a highly motivated patient with severe pulmonary disease in order to safely execute an unconventional approach to a conventional procedure.

\section{Acknowledgements}

None.

\section{Conflict of interest}

The author declares no conflict of interest.

\section{References}

1. Sinha R, Gurwara AK, Gupta SC. Laparoscopic surgery using spinal anesthesia. JSLS. 2008;12(2):133-138.

2. Sinha R, Gurwara AK, Gupta SC. Laparoscopic cholecystectomy under spinal anesthesia: a study of 3492 patients. J Laparoendosc Adv Surg Tech A. 2009;19(3):323-327.

3. Das W, Bhattacharya S, Ghosh S, et al. Comparison between general anesthesia and spinal anesthesia in attenuation of stress response in laparoscopic cholecystectomy: a randomized prospective trial. Saudi J Anaesth. 2015;9(2):184-188.

4. Kar M, Kar JK, Debnath B. Experience of laparoscopic cholecystectomy under spinal anesthesia with low-pressure pneumoperitoneum - prospective study of 300 Cases. Saudi J Gastroenterol. 2011;17(3):203-207.

5. van Zundert AA, Stultiens G, Jakimowicz JJ, et al. Segmental spinal anesthesia for cholecystectomy in a patient with severe lungs disease. $\mathrm{Br} J$ Anaesth. 2006;96(4):464-466.

6. Pursnani KG, Bazza Y, Calleja M, et al. Laparoscopic cholecystectomy under epidural anesthesia in patients with chronic respiratory disease. Surg Endosc. 1998;12(8):1082-1084.

7. Iafrati MD, Yarnell R, Schwaitzberg SD. Gasless laparoscopic cholecystectomy in pregnancy. J Laparoendosc Surg. 1995;5(2):127-130. 